\title{
Global attractors for Kirchhoff wave equation with nonlinear damping and memory
}

\author{
Suli Zhang ${ }^{1 *}$ (D) Jianwen Zhang ${ }^{2}$ and Haiyan Wang ${ }^{3}$
}

\section{"Correspondence:}

zhangsuli20100970@163.com

'College of Mechanical and Vehicle

Engineering, Taiyuan University of

Technology, Taiyuan, China

Full list of author information is

available at the end of the article

\begin{abstract}
In this paper, we prove that the existence of global attractors for a Kirchhoff wave equation with nonlinear damping and memory.
\end{abstract}

Keywords: Memory; Nonlinear damping; Global attractors

\section{Introduction}

Let $\Omega$ be an open bounded subset of $\mathbb{R}^{N}$ with sufficiently smooth boundary $\Gamma$, we consider the following Kirchhoff wave equation with nonlinear damping and linear memory:

$$
\begin{aligned}
& u_{t t}-M\left(\|\nabla u\|^{2}\right) \Delta u+a(x) g\left(u_{t}\right)-k(0) \Delta u-\int_{0}^{\infty} k^{\prime}(s) \Delta u(t-s) d s \\
& \quad+f(u)=h(x), \quad \text { in } \Omega \times \mathbb{R}^{+}, \\
& \left.u\right|_{x \in \Gamma}(x, t)=0, \quad u(x, 0)=u_{0}(x), \quad u_{t}(x, 0)=u_{1}(x), \\
& u(x, t)=u_{0}(x, t), \quad x \in \Omega, t \leq 0,
\end{aligned}
$$

where $M(s)=1+s^{\frac{m}{2}}, m \geq 1, k(0), k(\infty)>0$ and $k^{\prime}(s) \leq 0$ for every $s \in \mathbb{R}^{+}$, and the assumptions on nonlinear functions $f(u), g\left(u_{t}\right), a(x)$ and external force term $h(x)$ will be specified later.

This kind of wave models goes back to Kirchhoff. In 1883, Kirchhoff [1] firstly introduced the following equation to describe small vibrations of an elastic stretch string:

$$
u_{t t}-M\left(\|\nabla u\|^{2}\right) \Delta u=h,
$$

where $M(s)=a+b s$. There has been much research on global attractors; Lazo studied the existence for the IBVP of the Kirchhoff equation with memory term [2]

$$
u_{t t}-M\left(\|\nabla u\|^{2}\right) \Delta u+\int_{0}^{t} g(t-\tau) \Delta u(x, \tau) d \tau=0 .
$$

(c) The Author(s) 2020. This article is licensed under a Creative Commons Attribution 4.0 International License, which permits use, sharing, adaptation, distribution and reproduction in any medium or format, as long as you give appropriate credit to the original author(s) and the source, provide a link to the Creative Commons licence, and indicate if changes were made. The images or other third party material in this article are included in the article's Creative Commons licence, unless indicated otherwise in a credit line to the material. If material is not included in the article's Creative Commons licence and your intended use is not permitted by statutory regulation or exceeds the permitted use, you will need to obtain permission directly from the copyright holder. To view a copy of this licence, visit http://creativecommons.org/licenses/by/4.0/. 
Chueshov [3] studied the well-posedness and the global attractors of the Kirchhoff equation with strong nonlinear damping

$$
u_{t t}-\sigma\left(\|\nabla u\|^{2}\right) \Delta u_{t}-\phi\left(\|\nabla u\|^{2}\right) \Delta^{\theta} u+g(u)=h(x), \quad \frac{1}{2} \leq \theta<1 .
$$

Next, Chueshov [4] also studied the Kirchhoff equation with strong nonlinear damping in nature space $\mathcal{H}=H_{0}^{1}(\Omega) \cap L^{p+1}(\Omega) \times L^{2}(\Omega)$ as $\theta=1$. For related work on the Kirchhoff wave equations with strong damping, see $[5,6]$ and the references therein.

When $M(s)=0$, Eq. (1) become the well-known wave equation. Ma and Zhong [7] showed the existence of global attractors for the hyperbolic equation with memory

$$
u_{t t}+\alpha u_{t}-K(0) \Delta u-\int_{0}^{\infty} K^{\prime}(s) \Delta u(t-s) d s+g(u)=f .
$$

Recently, Park and Kang [8] studied the existence of global attractors for the semilinear hyperbolic with nonlinear damping and memory

$$
u_{t t}+a(x) g\left(u_{t}\right)+\lambda u-K(0) \Delta u-\int_{0}^{\infty} K^{\prime}(s) \Delta u(t-s) d s+f(u)=h(x) .
$$

In [9], Kang and Rivera showed the existence of global attractors for the beam equation localized nonlinear damping and memory

$$
u_{t t}+a(x) g\left(u_{t}\right)+\Delta^{2} u-K(0)\left(1+\|\nabla u\|^{2}\right) \Delta u-\int_{0}^{\infty} K^{\prime}(s) \Delta u(t-s) d s+f(u)=h .
$$

Motivated by [5, 7-9], we will prove the existence of global attractors for Eq. (1).

Following the framework proposed in [7], we shall add a new variable $\eta$ to the system, which corresponds to the relative displacement history. Let us define

$$
\eta=\eta^{t}(x, s)=u(x, t)-u(x, t-s) .
$$

By differentiation, we have

$$
\eta_{t}^{t}(x, s)=-\eta_{s}^{t}(x, s)+u_{t}(x, t)
$$

Let $\mu(s)=-k^{\prime}(s), k(\infty)=1,(1)$ transforms into the following system:

$$
\begin{aligned}
& u_{t t}-\left(1+\|\nabla u\|^{m}\right) \Delta u+a(x) g\left(u_{t}\right)-\int_{0}^{\infty} \mu(s) \Delta \eta(x, s) d s+f(u)=h, \\
& \eta_{t}=-\eta_{s}+u_{t},
\end{aligned}
$$

with boundary condition

$$
u=0, \quad \text { on } \Gamma \times \mathbb{R}^{+}, \quad \eta=0, \quad \text { on } \partial \Omega \times \mathbb{R}^{+} \times \mathbb{R}^{+},
$$

and initial conditions

$$
u(x, 0)=u_{0}(x), \quad u_{t}(x, 0)=u_{1}(x), \quad \eta^{t}(x, 0)=0, \quad \eta^{0}(x, s)=\eta_{0}(x, s) .
$$


This paper is organized as follows. In Sect. 2, we introduce some preliminaries. In Sect. 3, we show the existence of a bounded absorbing set in $\mathcal{H}$. In Sect. 4 , we give the existence of global attractors of problems (6)-(9).

\section{Preliminaries}

We first state some assumptions, which will be used in this paper.

Assumption (1) The memory kernel $\mu$ is required to satisfy the following hypotheses:

(h1) $\mu(s) \in C^{1}(\mathbb{R}) \cap L^{1}(\mathbb{R}), \forall s \in \mathbb{R}^{+}$;

(h2) $\int_{0}^{\infty} \mu(s) d s=k(0)$;

(h3) $\mu(s) \geq 0, \mu^{\prime}(s) \leq 0$;

(h4) $\mu^{\prime}(s)+k_{1} \mu(s) \leq 0, \forall s \in \mathbb{R}^{+}$, for some $k_{1}>0$.

Assumption (2) The function $a(x)$ satisfies

$$
a(x) \in L^{\infty}(\Omega), \quad a(x) \geq \alpha_{0}>0
$$

where $\alpha_{0}$ is a constant.

Assumption (3) The function $f \in C^{1}(\mathbb{R})$ satisfies

$$
\begin{gathered}
\left|f^{\prime}(s)\right| \leq C_{1}\left(1+|s|^{p}\right), \\
\lim _{|s| \rightarrow \infty} \inf \frac{f(s)}{s}>-\lambda_{1},
\end{gathered}
$$

where $0<p<\infty$, if $n \leq 2$, and $0<p \leq \frac{2}{n-2}$ if $n \leq 2 . \lambda_{1}$ is the constant in the Poincáre type inequality $\|\nabla u\|^{2} \geq \lambda_{1}\|u\|^{2}$.

Assumption (4) The damping function $g \in C^{1}(\mathbb{R})$ satisfies

$$
\begin{aligned}
& g(0)=0, \quad g \text { is strictly increasing, and } \liminf _{|s| \rightarrow \infty} g^{\prime}(s)>0, \\
& |g(s)| \leq C_{2}\left(1+|s|^{q}\right),
\end{aligned}
$$

with $1 \leq q<\infty$ if $n \leq 2$, and $1 \leq q \leq \frac{n+2}{n-2}$ if $n>2$.

In order to consider the relative displacement $\eta$ as a new variable, we introduce the weighted $L^{2}$-space

$$
\mathcal{M}=L_{\mu}^{2}\left(\mathbb{R}^{+} ; H_{0}^{1}\right)=\left\{\xi: \mathbb{R}^{+} \rightarrow H_{0}^{1}(\Omega) \mid \int_{0}^{\infty} \mu(s)\|\nabla \xi(s)\|_{2}^{2} d s<\infty\right\}
$$

which is a Hilbert space endowed with inner product and norm

$$
(\xi, \zeta)_{\mathcal{M}}=\int_{0}^{\infty} \mu(s)\left(\int_{\Omega} \nabla \xi(s) \nabla \zeta(s) d x\right) d s \quad \text { and } \quad\|\xi\|_{\mathcal{M}}^{2}=\int_{0}^{\infty} \mu(s)\|\nabla \xi\|_{2}^{2} d s
$$

respectively. 
Our analysis is given on the phase space

$$
\mathcal{H}=H_{0}^{1}(\Omega) \times L^{2}(\Omega) \times \mathcal{M}
$$

which is equipped with the norm

$$
\|(u, v, \eta)\|_{\mathcal{H}}^{2}=\|\nabla u\|^{2}+\|v\|^{2}+\|\eta\|_{\mathcal{M}}^{2} .
$$

In order to obtain the global attractors of the problems (6)-(9), we need the following theorem of existence, uniqueness of solution and continuous dependence on the initial data.

Theorem 2.1 ([9]) Let assumptions (1)-(4) hold, if $z_{0}=\left(u_{0}, v_{0}, \eta_{0}\right) \in \mathcal{H}$, then there exists a unique solution $z=\left(u, u_{t}, \eta\right)$ of $(6)-(9)$ such that

$$
z \in C([0, T], \mathcal{H}) \text { for all } T>0 \text {. }
$$

Next,we recall the simple compactness criterion stated in $[9,10]$.

Definition 2.1 ([9, 10]) Let $X$ be a Banach space and $B$ be a bounded subset of $X$, we call a function $\Phi(\cdot, \cdot)$ which defined on $X \times X$, is a contractive on $B \times B$ if for any sequence $\left\{x_{n}\right\}_{n=1}^{\infty} \subset B$, there is a subsequence $\left\{x_{n_{k}}\right\}_{k=1}^{\infty} \subset\left\{x_{n}\right\}_{n=1}^{\infty}$ such that

$$
\lim _{k \rightarrow \infty} \lim _{l \rightarrow \infty} \Phi_{T}\left(x_{n_{k}}, x_{n_{l}}\right)=0
$$

Denote all such contractive functions on $B \times B$ by $C(B)$.

Theorem $2.2([9,10])$ Let $\{s(t)\}_{t \geq 0}$ be a semigroup on a Banach space $(X,\|\cdot\|)$ and has a bounded absorbing set $B_{0}$. Moreover, assume that for any $\varepsilon \geq 0$ there exist $T=T\left(B_{0}, \varepsilon\right)$ and $\Phi(\cdot, \cdot) \in C(B)$ such that

$$
\|S(T) x-S(T) y\| \leq \varepsilon+\Phi_{T}(x, y) \quad \text { for all } x, y \in B_{0},
$$

where $\Phi_{T}$ depends on $T$. Then $\{s(t)\}_{t \geq 0}$ is asymptotically compact in $X$, i.e., for any bounded sequence $\left\{y_{n}\right\}_{n}^{\infty} \subset X$ and $\left\{t_{n}\right\}$ with $t_{n} \rightarrow \infty,\left\{S\left(t_{n}\right) y_{n}\right\}_{n=1}^{\infty}$ is compact in $X$.

Lemma 2.1 ([11]) Let $g(\cdot)$ satisfy condition (13). Then for any $\delta>0$ there exists $c(\delta)>0$, such that

$$
|u-v|^{2} \leq \delta+C(\delta)(g(u)-g(v))(u-v), \quad \text { for all } u, v \in \mathbb{R} .
$$

\section{Absorbing set in $\mathcal{H}$}

In this section, we prove the existence of the bounded absorbing set in $\mathcal{H}$. We use $C_{i}$ to denote several positive constants.

Lemma 3.1 Under assumptions (1)-(4), the semigroup $\{S(t)\}_{t \geq 0}$ corresponding to problems (6)-(9) has a bounded absorbing set in $\mathcal{H}$. 
Proof we take the scalar product in $L^{2}$ of system (6) with $u_{t}$ and (7) with $\eta$, respectively, we have

$$
\begin{aligned}
& \frac{d}{d t}\left(\frac{1}{2}\left\|u_{t}\right\|^{2}+\frac{1}{2}\|\nabla u\|^{2}+\frac{1}{m+2}\|\nabla u\|^{m+2}+\frac{1}{2}\|\eta\|_{\mathcal{M}}^{2}+\int_{\Omega}(F(u)-h u) d x\right) \\
& \quad+\left(\eta, \eta_{s}\right)_{\mathcal{M}}+\left(a(x) g\left(u_{t}\right), u_{t}\right)=0,
\end{aligned}
$$

where $F(u)=\int_{0}^{u} f(s) d s$. As in [7]

$$
\begin{aligned}
\left(\eta, \eta_{s}\right)_{\mathcal{M}} & =\frac{1}{2} \int_{0}^{\infty} \mu(s) \frac{d}{d s}\|\nabla \eta(s)\|^{2} d s \\
& =-\frac{1}{2} \int_{0}^{\infty} \mu^{\prime}(s)\|\nabla \eta(s)\|^{2} d s \geq \frac{k_{1}}{2}\|\eta\|_{\mathcal{M}}^{2} .
\end{aligned}
$$

We set

$$
E(t)=\frac{1}{2}\left\|u_{t}\right\|^{2}+\frac{1}{2}\|\nabla u\|^{2}+\frac{1}{m+2}\|\nabla u\|^{m+2}+\frac{1}{2}\|\eta\|_{\mathcal{M}}^{2}+\int_{\Omega}(F(u)-h u) d x .
$$

Then from (17) and (18) we obtain

$$
\frac{d}{d t} E(t)+\frac{k_{1}}{2}\|\eta\|_{\mathcal{M}}^{2}+\int_{\Omega}\left(a(x) g\left(u_{t}\right) u_{t}\right) d x \leq 0
$$

From (10), (13) we obtain

$$
E(t) \leq E(0), \quad t \geq 0
$$

By the hypothesis (12) we know that there are $\lambda>\lambda_{1}>0$ and $C_{0}$ such that

$$
(f(u), u)>-\frac{\lambda}{2}\|u\|^{2}-C_{0} \operatorname{mes}(\Omega), \quad \int_{\Omega} F(u) d x>-\frac{\lambda}{4}\|u\|^{2}-C_{0} \operatorname{mes}(\Omega) .
$$

Using the Young inequality, we have

$$
-\int_{\Omega} h u d x \geq-\varepsilon\|u\|^{2}-\frac{1}{4 \varepsilon}\|h\|^{2}
$$

we choose proper $\lambda$ and $\varepsilon$ small enough so that $\frac{1}{2}-\frac{\lambda}{4 \lambda_{1}}-\varepsilon>\frac{1}{8}$, and we have

$$
\begin{aligned}
E(0) & \geq E(t) \geq \frac{1}{2}\left\|u_{t}\right\|^{2}+\frac{1}{m+2}\|\nabla u\|^{m+2}+\frac{1}{8}\|\nabla u\|^{2}+\frac{1}{2}\|\eta\|_{\mathcal{M}}^{2}-C_{1}\left(\operatorname{mes}(\Omega)+\|h\|^{2}\right) \\
& \geq-C_{1}\left(\operatorname{mes}(\Omega)+\|h\|^{2}\right),
\end{aligned}
$$

combining (19) with (22), we have

$$
\int_{0}^{t} \int_{\Omega}\left(a(x) g\left(u_{t}\right) u_{t}\right) d x \leq E(0)-E(t) \leq E(0)+C_{1}\left(\operatorname{mes}(\Omega)+\|h\|^{2}\right), \quad \forall t \geq 0 .
$$


Taking the scalar product in $L^{2}$ of (6) with $v=u_{t}+\varepsilon u$, we obtain

$$
\begin{aligned}
& \frac{d}{d t}\left(\frac{1}{2}\|v\|^{2}+\frac{1}{2}\|\nabla u\|^{2}+\frac{1}{m+2}\|\nabla u\|^{m+2}+\frac{1}{2}\|\eta\|_{\mathcal{M}}^{2}-\frac{\varepsilon^{2}}{2}\|u\|^{2}+\varepsilon\|\nabla u\|^{2}\right. \\
& \left.\quad+\int_{\Omega}(F(u)-h u) d x\right)+\varepsilon\|\nabla u\|^{m+2}+\frac{k_{1}}{2}\|\eta\|_{\mathcal{M}}^{2}+\varepsilon(f(u), u) \\
& \quad+\left(a(x) g\left(u_{t}\right)-\varepsilon u_{t}, u_{t}\right)+\varepsilon\left(a(x) g\left(u_{t}\right), u\right)-\varepsilon(h, u) \leq \varepsilon(\eta, u)_{\mathcal{M}}
\end{aligned}
$$

Let

$$
\begin{aligned}
F(t)= & \frac{1}{2}\|v\|^{2}+\frac{1}{2}\|\nabla u\|^{2}+\frac{1}{m+2}\|\nabla u\|^{m+2}+\frac{1}{2}\|\eta\|_{\mathcal{M}}^{2}-\frac{\varepsilon^{2}}{2}\|u\|^{2}+\int_{\Omega}(F(u)-h u) d x, \\
G(t)= & \varepsilon\|\nabla u\|^{2}+\varepsilon\|\nabla u\|^{m+2}+\frac{k_{1}}{2}\|\eta\|_{\mathcal{M}}^{2}+\varepsilon(f(u), u)-\varepsilon(h, u)-\varepsilon(\eta, u)_{\mathcal{M}} \\
& +\left(a(x) g\left(u_{t}\right)-\varepsilon u_{t}, u_{t}\right)+\varepsilon\left(a(x) g\left(u_{t}\right), u\right),
\end{aligned}
$$

So

$$
\frac{d}{d t} F(t)+G(t) \leq 0
$$

Similarly, using (21), the Poincáre inequality and the Young inequality, choosing proper $\lambda$ and $\varepsilon$ small enough so that $\frac{1}{2}-\frac{\varepsilon^{2}}{2 \lambda_{1}}-\frac{\lambda}{4 \lambda_{1}}-\varepsilon>\frac{1}{8}$, we have

$$
F(t) \geq \frac{1}{2}\|v\|^{2}+\frac{1}{8}\|\nabla u\|^{2}+\frac{1}{m+2}\|\nabla u\|^{m+2}+\frac{1}{2}\|\eta\|_{\mathcal{M}}^{2}-C\left(\operatorname{mes}(\Omega)+\|h\|^{2}\right) .
$$

It is obvious that (10) and (13) imply that there are $\varepsilon>0$ and $C>0$ such that

$$
\begin{aligned}
& \left(a(x) g\left(u_{t}\right), u_{t}\right) \geq 2 \varepsilon\left\|u_{t}\right\|^{2}-C_{\varepsilon} \operatorname{mes}(\Omega), \\
& \left(a(x) g\left(u_{t}\right)-\varepsilon u, u_{t}\right) \geq \varepsilon\left\|u_{t}\right\|^{2}-C(\varepsilon) \operatorname{mes}(\Omega) .
\end{aligned}
$$

Due to the Young inequality we have

$$
\varepsilon(\eta, u)_{\mathcal{M}} \geq-\frac{k_{1}}{4}\|\varepsilon\|_{\mathcal{M}}^{2}-\frac{k(0) \delta^{2}}{k_{1}}\|\nabla u\|^{2}
$$

Using (13) and (14) yields

$$
|g(s)|^{\frac{q+1}{q}}=|g(s)|^{\frac{1}{q}}|g(s)| \leq C(1+|s|)|g(s)|
$$

so

$$
\begin{cases}|g(s)|^{\frac{q+1}{q}} \leq C, & |s| \leq 1 \\ |g(s)|^{\frac{q+1}{q}} \leq 2 C g(s) s, & |s| \geq 1\end{cases}
$$

where $C$ is a constant which is independent of $s$. 
Then from (29), using the Hölder inequality, the Young inequality and the Sobolev embedding $H_{0}^{1}(\Omega) \hookrightarrow L^{q+1}(\Omega)$, we obtain

$$
\begin{aligned}
\left|\int_{\Omega} a(x) g\left(u_{t}\right) u d x\right| & \\
\leq & \int_{\Omega\left(\left|u_{t}\right| \leq 1\right)}\left|a(x) g\left(u_{t}\right) u\right| d x+\int_{\Omega\left(\left|u_{t}\right| \geq 1\right)}\left|a(x) g\left(u_{t}\right) u\right| d x \\
\leq & \int_{\Omega\left(\left|u_{t}\right| \leq 1\right)} C|a(x) u| d x \\
& +\left(\int_{\Omega\left(\left|u_{t}\right| \geq 1\right)} a(x)\left|g\left(u_{t}\right)\right|^{\frac{q+1}{q}} d x\right)^{\frac{q}{q+1}}\left(\int_{\Omega\left(\left|u_{t}\right| \geq 1\right)} a(x)|u|^{q+1} d x\right)^{\frac{1}{q+1}} \\
\leq & \int_{\Omega\left(\left|u_{t}\right| \leq 1\right)} C|a(x) u| d x \\
& +2 C\left(\int_{\Omega\left(\left|u_{t}\right| \geq 1\right)} a(x) g\left(u_{t}\right) u_{t} d x\right)^{\frac{q}{q+1}}\left(\int_{\Omega\left(\left|u_{t}\right| \geq 1\right)} a(x)|u|^{q+1} d x\right)^{\frac{1}{q+1}} \\
\leq & \frac{C}{4 \gamma} \int_{\Omega}\left|\frac{a(x)}{a_{0}}\right|^{2} d x \\
& +C \gamma a_{0}^{2}\|u\|^{2}+C_{\gamma}\left(\int_{\Omega\left(\left|u_{t}\right| \geq 1\right)} a(x) g\left(u_{t}\right) u_{t} d x\right)\|u\|_{q+1}^{q}+\eta\|u\|_{q+1}^{2} \\
\leq & \frac{C}{4 \gamma} \operatorname{mes}(\Omega)+C \gamma a_{0}^{2}\|u\|^{2}+C_{s} C_{\gamma}\|\nabla u\|^{\frac{q-1}{q}} \int_{\Omega} a(x) g\left(u_{t}\right) u_{t} d x+\gamma C_{s}\|\nabla u\|^{2},
\end{aligned}
$$

where $a_{0}=\sup _{x \in \Omega} a(x)$, and $\gamma$ is a constant. From (21), (27), (28), (30) we have

$$
\begin{aligned}
G(t) \geq & \varepsilon\left\|u_{t}\right\|^{2}+\varepsilon\|\nabla u\|^{m+2}+\frac{k_{1}}{4}\|\eta\|_{\mathcal{M}}^{2} \\
& +\varepsilon\left(\frac{1}{2}-\frac{k(0) \varepsilon^{2}}{k_{1}}-C\right)\|\nabla u\|^{2}-\left(\varepsilon C \gamma a_{0}^{2}+\frac{\varepsilon \lambda}{4}\right)\|u\|^{2} \\
& -\varepsilon C\|\nabla u\|^{\frac{q-1}{q}} \int_{\Omega} a(x) g\left(u_{t}\right) u_{t} d x-C_{\varepsilon}^{\prime}\left(\operatorname{mes}(\Omega)+\|h\|^{2}\right),
\end{aligned}
$$

we choose $\varepsilon$ and $C$ small enough so that $\frac{1}{2}-\frac{k(0) \varepsilon^{2}}{k_{1}}-C>\frac{1}{4}$, we get

$$
\begin{aligned}
G(t) \geq & \frac{\varepsilon}{4}\left(\left\|u_{t}\right\|^{2}+\|\nabla u\|^{2}\right)+\frac{k_{1}}{4}\|\eta\|_{\mathcal{M}}^{2} \\
& -C_{E(0)} \int_{\Omega} a(x) g\left(u_{t}\right) u_{t} d x-C_{\varepsilon}^{\prime}\left(\operatorname{mes}(\Omega)+\|h\|^{2}\right),
\end{aligned}
$$

where $C_{E(0)}$ is a constant which depends on $\varepsilon, \gamma, \mathrm{C}$ and $E(0), C_{\varepsilon}^{\prime}$ is a constant depending on $\varepsilon, C_{\delta}$ and $\mathrm{C}$.

We have

$$
\left\|u_{t}\right\|^{2}+\|\nabla u\|^{2}+\|\eta\|_{\mathcal{M}}^{2}=\left\|u_{t}+\delta u-\delta u\right\|^{2}+\|\nabla u\|^{2}+\|\eta\|_{\mathcal{M}}^{2}
$$




$$
\begin{aligned}
& \leq 2\|v\|^{2}+\left(\frac{2 \delta^{2}}{\lambda_{1}}+1\right)\|\nabla u\|^{2}+\|\eta\|_{\mathcal{M}}^{2} \\
& \leq C_{0}\left(\|v\|^{2}+\|\nabla u\|^{2}+\|\eta\|_{\mathcal{M}}^{2}\right),
\end{aligned}
$$

where $C_{0}=\max \left\{2,1+\frac{2 \delta^{2}}{\lambda_{1}}\right\}$.

Integrating (25), combining with (23), (26), (31), yields

$$
\begin{aligned}
\left\|u_{t}\right\|^{2} & +\|\nabla u\|^{2}+\|\eta\|_{\mathcal{M}}^{2}-4 C\left(\operatorname{mes}(\Omega)+\|h\|^{2}\right) \\
& -4 C_{0} F(0)-4 C_{0} C_{E(0)}\left(E(0)+C\left(\operatorname{mes}(\Omega)+\|h\|^{2}\right)\right. \\
\leq & -\int_{0}^{t}\left(\delta^{\prime} C_{0}\left(\left\|u_{t}(s)\right\|^{2}+\|\nabla u(s)\|^{2}+\left\|\eta^{s}(\tau)\right\|_{\mathcal{M}}^{2}\right)\right. \\
& \left.-4 C_{0} C_{\varepsilon}^{\prime}\left(\operatorname{mes}(\Omega)+\|h\|^{2}\right)\right) d s,
\end{aligned}
$$

where $\delta^{\prime}=\min \left\{\delta, k_{1}\right\}$. Therefore, for any $\rho>\frac{4 C_{\varepsilon}^{\prime}\left(\operatorname{mes}(\Omega)+\|h\|^{2}\right)}{\delta^{\prime}}$ there exists $t_{0}$ such that

$$
\left\|u_{t}\left(t_{0}\right)\right\|^{2}+\left\|\nabla u\left(t_{0}\right)\right\|^{2}+\left\|\eta^{t_{0}}(\tau)\right\|_{\mathcal{M}}^{2} \leq \rho .
$$

Set

$$
B_{0}=\left\{\left(u_{0}, v_{0}, \eta_{0}\right) \in \mathcal{H} \mid\left\|\nabla u_{0}\right\|^{2}+\left\|v_{0}\right\|^{2}+\left\|\eta_{0}\right\|_{\mathcal{M}}^{2} \leq \rho\right\},
$$

then we see $B_{0}$ is a bounded absorbing set. Define

$$
B_{1}=\bigcup_{t \geq 0} S(t) B_{0}
$$

so $B_{1}$ is also a bounded absorbing set.

\section{Existence of the global attractor in $\mathcal{H}$}

\subsection{A priori estimate}

Firstly, we use the prior estimates to obtain the asymptotic compactness following the standard energy method. In this section, $C_{i}$ are positive constants.

Let $\left(u, u_{t}, \eta\right)$ and $\left(v, v_{t}, \xi\right)$ be two solution to systems (6)-(9), and $\left(u, u_{t}, \eta\right)$ and $\left(v, v_{t}, \xi\right) \in$ $B_{1}, \omega(t)=u(t)-v(t), \zeta=\eta-\xi$. Then $\omega(t), \zeta$ satisfy

$$
\begin{aligned}
& \omega_{t t}-\|\nabla u\|^{m} \Delta u+\|\nabla v\|^{m} \Delta v-\Delta \omega-\int_{0}^{\infty} \mu(s) \Delta \zeta(s) d s \\
& \quad+a(x) g\left(u_{1 t}\right)-a(x) g\left(u_{2 t}\right)+f\left(u_{1}\right)-f\left(u_{2}\right)=0, \\
& \zeta_{t}=-\zeta_{s}+\omega_{t},
\end{aligned}
$$


firstly, taking the scalar product in $L^{2}$ of (35) with $\omega$ and integrating over [0,T], we get

$$
\begin{aligned}
\int_{0}^{T}\|\nabla \omega(s)\|^{2} d s= & \int_{\Omega} \omega_{t}(0) \omega(0) d x-\int_{\Omega} \omega_{t}(T) \omega(T) d x+\int_{0}^{T}\left\|\omega_{t}(s)\right\|^{2} d s \\
& -\int_{0}^{T}\|\nabla u(s)\|^{m}\|\nabla \omega(s)\|^{2} d s-\int_{0}^{T}(\zeta, \omega)_{\mathcal{M}} d s \\
& -\int_{0}^{T} \int_{\Omega}\left(\|\nabla u(s)\|^{m}-\|\nabla v(s)\|^{m}\right) \nabla v(s) \nabla \omega(s) d x d s \\
& -\int_{0}^{T} \int_{\Omega} a(x)\left(g\left(u_{t}(s)\right)-g\left(v_{t}(s)\right)\right) \omega(s) d x d s \\
& -\int_{0}^{T} \int_{\Omega}(f(u(s))-f(v(s))) \omega(s) d x d s .
\end{aligned}
$$

Using the Young inequality and $(h 3)$, we obtain

$$
(\zeta, \omega)_{\mathcal{M}} \geq-\frac{1}{2}\|\nabla \omega\|^{2}-\frac{k(0)}{2}\|\zeta\|_{\mathcal{M}}^{2} .
$$

Secondly, taking the scalar product in $L^{2}$ of (35), (36) with $\omega_{t}$ and integrating over $[0, T]$, we get

$$
\begin{array}{r}
\frac{d}{d t}\left(\frac{1}{2}\left\|\omega_{t}\right\|^{2}+\frac{1}{2}\|\nabla \omega\|^{2}+\frac{1}{2}\|\zeta\|_{\mathcal{M}}^{2}\right)+\int_{\Omega}\left(\|\nabla u\|^{m}-\|\nabla v\|^{m}\right) \nabla v \nabla \omega_{t} d x \\
+\left(\zeta, \zeta_{s}\right)_{\mathcal{M}}+\int_{\Omega}(f(u)-f(v)) \omega_{t} d x+\int_{\Omega} a(x)\left(g\left(u_{t}\right)-g\left(v_{t}\right)\right) \omega_{t} d x=0 .
\end{array}
$$

Let

$$
E_{\omega}(t)=\frac{1}{2}\left\|\omega_{t}\right\|^{2}+\frac{1}{2}\|\nabla \omega\|^{2}+\frac{1}{2}\|\zeta\|_{\mathcal{M}}^{2} .
$$

Integrating (39) over $(s, T]$ and combining with (38), where $s \in[0, T]$, we have

$$
\begin{aligned}
E_{\omega}(t) & +\frac{k_{1}}{2} \int_{s}^{T}\|\zeta\|_{\mathcal{M}}^{2}+\int_{s}^{T} \int_{\Omega} a(x)\left(g\left(u_{t}(\tau)\right)-g\left(v_{t}(\tau)\right)\right) \omega_{t}(\tau) d x d \tau \\
& +\frac{1}{2} \int_{\Omega}\|\nabla u(T)\|^{m}\|\nabla \omega(T)\|^{2} d x \\
\leq & E_{\omega}(s)+\frac{1}{2} \int_{\Omega}\|\nabla u(s)\|^{m}\|\nabla \omega(s)\|^{2} d x \\
& +\frac{m}{2} \int_{s}^{T} \int_{\Omega}\|\nabla \omega(\tau)\|^{2}\|\nabla u(\tau)\|^{m-1} \nabla u_{t}(\tau) d x d \tau \\
& -\int_{s}^{T} \int_{\Omega}\left(\|\nabla u(\tau)\|^{m}-\|\nabla v(\tau)\|^{m}\right) \nabla v(\tau) \nabla \omega_{t}(\tau) d x d \tau \\
& -\int_{s}^{T} \int_{\Omega}(f(u(\tau))-f(v(\tau))) \omega_{t}(\tau) d x d \tau .
\end{aligned}
$$


Integrating (40) over $[0, T]$ with respect to $s$, we get

$$
\begin{aligned}
T E_{\omega}(t) \leq & \int_{0}^{T} E_{\omega}(s) d s+\frac{1}{2} \int_{0}^{T} \int_{\Omega}\|\nabla u(s)\|^{m}\|\nabla \omega(s)\|^{2} d x \\
& +\frac{m}{2} \int_{0}^{T} \int_{s}^{T} \int_{\Omega}\|\nabla \omega(\tau)\|^{2}\|\nabla u(\tau)\|^{m-1} \nabla u_{t}(\tau) d x d \tau d s \\
& -\int_{0}^{T} \int_{s}^{T} \int_{\Omega}\left(\|\nabla u(\tau)\|^{m}-\|\nabla v(\tau)\|^{m}\right) \nabla v(\tau) \nabla \omega_{t}(\tau) d x d \tau d s \\
& -\int_{0}^{T} \int_{s}^{T} \int_{\Omega}(f(u(\tau))-f(v(\tau))) \omega_{t}(\tau) d x d \tau d s .
\end{aligned}
$$

Due to (10), (40), and Lemma 2.1, we obtain, for any $\delta>0$,

$$
\begin{aligned}
\int_{0}^{T}\left\|\zeta^{\tau}\right\|_{\mathcal{M}}^{2} d \tau+\int_{0}^{T}\left\|\omega_{t}(\tau)\right\|^{2} d \tau \\
\leq C_{2} E_{\omega}(0)-C_{2} \int_{0}^{T} \int_{\Omega}(f(u(\tau))-f(v(\tau))) \omega_{t}(\tau) d x d \tau \\
\quad+\delta T \operatorname{mes}(\Omega)-\frac{C_{2}}{2} \int_{\Omega}\|\nabla u(T)\|^{m}\|\nabla \omega(T)\|^{2} d x \\
\quad-C_{2} \int_{0}^{T} \int_{\Omega}\left(\|\nabla u(\tau)\|^{m}-\|\nabla v(\tau)\|^{m}\right) \nabla v(\tau) \nabla \omega_{t}(\tau) d x d \tau \\
\quad-C_{2} \int_{0}^{T} \int_{\Omega}(f(u(\tau))-f(v(\tau))) \omega_{t}(\tau) d x d \tau \\
+\frac{m C_{2}}{2} \int_{0}^{T} \int_{\Omega}\|\nabla \omega(\tau)\|^{2}\|\nabla u(\tau)\|^{m-1} \nabla u_{t}(\tau) d x d \tau
\end{aligned}
$$

where $C_{2}$ is a constant which depends on $\delta, \alpha_{0}$ and $k_{1}$.

Thus, from (37), (38) and (42) we have

$$
\begin{aligned}
\int_{0}^{T} E_{\omega}(t) d t \leq & C_{3} \delta T \operatorname{mes}(\Omega)+C_{2} C_{3} E_{\omega}(0)-\frac{C_{2} C_{3}}{2} \int_{\Omega}\|\nabla u(T)\|^{m}\|\nabla \omega(T)\|^{2} d x \\
& +\frac{C_{2} C_{3}}{2} \int_{\Omega}\|\nabla u(0)\|^{m}\|\nabla \omega(0)\|^{2} d x \\
& -C_{2} C_{3} \int_{0}^{T} \int_{\Omega}\left(\|\nabla u(\tau)\|^{m}-\|\nabla v(\tau)\|^{m}\right) \nabla v(\tau) \nabla \omega_{t}(\tau) d x d \tau \\
& -C_{2} C_{3} \int_{0}^{T} \int_{\Omega}(f(u(\tau))-f(v(\tau))) \omega_{t}(\tau) d x d \tau \\
& +\frac{m C_{2} C_{3}}{2} \int_{0}^{T} \int_{\Omega}\|\nabla \omega(\tau)\|^{2}\|\nabla u(\tau)\|^{m-1} \nabla u_{t}(\tau) d x d \tau \\
& +\int_{\Omega}^{\omega_{t}(0) \omega(0) d x-\int_{\Omega} \omega_{t}(T) \omega(T) d x} \\
& -\int_{0}^{T}\|\nabla u(s)\|^{m}\|\nabla \omega(s)\|^{2} d s \\
& -\int_{0}^{T} \int_{\Omega}\left(\|\nabla u(s)\|^{m}-\|\nabla v(s)\|^{m}\right) \nabla v(s) \nabla \omega(s) d x d s
\end{aligned}
$$




$$
\begin{aligned}
& -\int_{0}^{T} \int_{\Omega} a(x)\left(g\left(u_{t}(s)\right)-g\left(v_{t}(s)\right)\right) \omega(s) d x d s \\
& -\int_{0}^{T} \int_{\Omega}(f(u(s))-f(v(s))) \omega(s) d x d s,
\end{aligned}
$$

where $C_{3}=\max \left\{\frac{3}{2}, \frac{k(0)+1}{2}\right\}$. From (23) and the existence of the absorbing set, we get

$$
\begin{aligned}
& \int_{0}^{T} \int_{\Omega} a(x)\left(g\left(u_{t}\right)\right) u_{t} d x d s \leq C_{\rho} \\
& \int_{0}^{T} \int_{\Omega} a(x)\left(g\left(v_{t}\right)\right) v_{t} d x d s \leq C_{\rho}
\end{aligned}
$$

where $C_{\rho}$ is a constant which depends on mes $(\Omega),\|h\|^{2}$ and the size of $B_{0}$. By a similar method to that of (30) and (43), (44), we have

$$
\begin{aligned}
& \left|\int_{0}^{T} \int_{\Omega} a(x) g\left(u_{t}(s)\right) \omega(s) d x d s\right| \\
& \leq C^{\frac{q}{q+1}} \int_{0}^{T} \int_{\Omega\left(\left\|u_{t}\right\| \leq 1\right)}|a(x) \omega| d x d s \\
& \quad+(2 C)^{\frac{q}{q+1}}\left(\int_{0}^{T} \int_{\Omega\left(\left|u_{t}\right| \geq 1\right)} a(x) g\left(u_{t}\right) u_{t} d x d s\right)^{\frac{q}{q+1}} \\
& \quad \times\left(\int_{0}^{T} \int_{\Omega\left(\left|u_{t}\right| \geq 1\right)} a(x)|\omega|^{q+1} d x d s\right)^{\frac{1}{q+1}} \\
& \leq C^{\frac{q}{q+1}} \int_{0}^{T} \int_{\Omega} a(x)|\omega| d x d s+C_{\rho} T^{\frac{1}{q+1}}
\end{aligned}
$$

similarly

$$
\left|\int_{0}^{T} \int_{\Omega} a(x) g\left(v_{t}(s)\right) \omega(s) d x d s\right| \leq C^{\frac{q}{q+1}} \int_{0}^{T} \int_{\Omega} a(x)|\omega| d x d s+C_{\rho} T^{\frac{1}{q+1}}
$$

combining (41), (43), (46), (47), we have

$$
T E_{\omega}(T) \leq C_{B}+\Phi_{T}\left(z_{0}^{1}, z_{0}^{2}\right)
$$

where

$$
\begin{aligned}
C_{B}= & C_{3} \delta T \operatorname{mes}(\Omega)+C_{2} C_{3} E_{\omega}(0)+\int_{\Omega} \omega_{t}(0) \omega(0) d x-\int_{\Omega} \omega_{t}(T) \omega(T) d x+2 C_{\rho} T^{\frac{1}{q+1}} \\
& +\frac{C_{2} C_{3}}{2} \int_{\Omega}\|\nabla u(0)\|^{m}\|\nabla \omega(0)\|^{2} d x-\frac{C_{2} C_{3}}{2} \int_{\Omega}\|\nabla u(T)\|^{m}\|\nabla \omega(T)\|^{2} d x,
\end{aligned}
$$




$$
\begin{aligned}
\Phi_{T}\left(z_{0}^{1}, z_{0}^{2}\right)= & -C_{2} C_{3} \int_{0}^{T} \int_{\Omega}\left(\|\nabla u(\tau)\|^{m}-\|\nabla v(\tau)\|^{m}\right) \nabla v(\tau) \nabla \omega_{t}(\tau) d x d \tau \\
& -C_{2} C_{3} \int_{0}^{T} \int_{\Omega}(f(u(\tau))-f(v(\tau))) \omega_{t}(\tau) d x d \tau \\
& +\frac{m C_{2} C_{3}}{2} \int_{0}^{T} \int_{\Omega}\|\nabla \omega(\tau)\|^{2}\|\nabla u(\tau)\|^{m-1} \nabla u_{t}(\tau) d x d \tau \\
& -\int_{0}^{T} \int_{\Omega}\left(\|\nabla u(s)\|^{m}-\|\nabla v(s)\|^{m}\right) \nabla v(s) \nabla \omega(s) d x d s \\
& -\int_{0}^{T} \int_{\Omega}(f(u(s))-f(v(s))) \omega(s) d x d s-\int_{0}^{T}\|\nabla u(s)\|^{m}\|\nabla \omega(s)\|^{2} d s \\
& +2 C^{\frac{q}{q+1}} \int_{0}^{T} \int_{\Omega} a(x)|\omega(s)| d x d s+\frac{1}{2} \int_{0}^{T} \int_{\Omega}\|\nabla u(s)\|^{m}\|\nabla \omega(s)\|^{2} d x d s \\
& +\frac{m}{2} \int_{0}^{T} \int_{s}^{T} \int_{\Omega}\|\nabla \omega(\tau)\|^{2}\|\nabla u(\tau)\|^{m-1} \nabla u_{t}(\tau) d x d \tau d s \\
& -\int_{0}^{T} \int_{s}^{T} \int_{\Omega}\left(\|\nabla u(\tau)\|^{m}-\|\nabla v(\tau)\|^{m}\right) \nabla v(\tau) \nabla \omega_{t}(\tau) d x d \tau d s \\
& -\int_{0}^{T} \int_{s}^{T} \int_{\Omega}(f(u(\tau))-f(v(\tau))) \omega_{t}(\tau) d x d \tau d s .
\end{aligned}
$$

Then we have

$$
E_{\omega}(T) \leq \frac{C_{B}}{T}+\frac{1}{T} \Phi_{T}\left(z_{0}^{1}, z_{0}^{2}\right) .
$$

\subsection{Asymptotic compactness}

In this subsection, following the argument in $[9,10]$, we will prove the asymptotic compactness of the semigroup $\{S(t)\}_{t \geq 0}$ in $\mathcal{H}$, which is given in the following theorem.

Theorem 4.1 Under assumptions (1)-(4), the semigroup $\{S(t)\}_{t \geq 0}$ to systems (6)-(9) is asymptotically compact in $\mathcal{H}$.

Proof since the semigroup $\{S(t)\}_{t \geq 0}$ has a bounded absorbing set, for every fixed $\varepsilon>0$, we can choose that $\delta \leq \frac{\varepsilon}{2 C_{3} \operatorname{mes}(\Omega)}$, and then let $T$ become so large that

$$
\frac{C_{B}}{T} \leq \varepsilon
$$

Hence, thanks to Theorem 2.2, we only need to verify that the function $\Phi_{T}\left(z_{0}^{1}, z_{0}^{2}\right)$ defined in (50) belongs to $C\left(B_{1}\right)$ for each fixed $T$. and we claim that

$$
\left\|S(t) z_{0}^{1}-S(t) z_{0}^{2}\right\|_{\mathcal{H}} \leq \varepsilon+\Phi_{T}\left(z_{0}^{1}, z_{0}^{2}\right), \quad \forall z_{0}^{1}, z_{0}^{2} \in B
$$

Here $\left(u(t), u_{t}(t), \eta\right)=S(t) z_{0}^{1}$ and $\left(v(t), v_{t}(t), \xi\right)=S(t) z_{0}^{1}$ are the solutions of (6)-(9) with respect to initial $z_{0}^{1}, z_{0}^{2} \in B_{1}$. Then, since $C\left(B_{1}\right)$ is a bounded positively invariant set in $\mathcal{H}$, it follows that $\left(u_{n}, u_{n_{t}}, \eta^{n}\right)$ is uniformly bounded in $\mathcal{H}$. We have

$$
u_{n} \rightarrow u \quad \text { weakly star in } L^{\infty}\left(0, T ; H_{0}^{1}(\Omega)\right)
$$




$$
u_{n_{t}} \rightarrow u_{t} \quad \text { weakly star in } L^{\infty}\left(0, T ; L^{2}(\Omega)\right)
$$

Then, by the compact embedding $H_{0}^{1}(\Omega) \hookrightarrow L^{k}(\Omega)$, we have

$$
\begin{aligned}
& u_{n} \rightarrow u \quad \text { strongly in } L^{2}\left(0, T ; L^{2}(\Omega)\right), \\
& u_{n} \rightarrow u \quad \text { strongly in } L^{k}\left(0, T ; L^{k}(\Omega)\right),
\end{aligned}
$$

where $k \leq \frac{2 n}{n-2}$, therefore from (56) we have

$$
\begin{aligned}
& \lim _{l \rightarrow \infty} \lim _{k \rightarrow \infty} \int_{0}^{T} \int_{\Omega}\left(f\left(u_{l}(\tau)\right)-f\left(u_{k}(\tau)\right)\right)\left(u_{l_{t}}(\tau)-u_{k_{t}}(\tau)\right) d x d \tau=0, \\
& \lim _{l \rightarrow \infty} \lim _{k \rightarrow \infty} \int_{0}^{T} \int_{\Omega}\left(f\left(u_{l}(\tau)\right)-f\left(u_{k}(\tau)\right)\right)\left(u_{l}(\tau)-u_{k}(\tau)\right) d x d \tau=0,
\end{aligned}
$$

then from (57) and (10), we obtain

$$
\lim _{l \rightarrow \infty} \lim _{k \rightarrow \infty} \int_{0}^{T} \int_{\Omega} a(x)\left|u_{l}(s)-u_{k}(s)\right| d x d s=0
$$

Finally, we follow a similar argument to the ones given in $[9,10]$. We have

$$
\begin{aligned}
& \lim _{l \rightarrow \infty} \lim _{k \rightarrow \infty} \int_{0}^{T} \int_{\Omega}\left\|\nabla u_{l}(\tau)-\nabla u_{k}(\tau)\right\|^{2} \nabla u_{l}(\tau) \nabla u_{k_{t}}(\tau) d x d \tau=0, \\
& \lim _{l \rightarrow \infty} \lim _{k \rightarrow \infty} \int_{0}^{T} \int_{\Omega}\left(\left\|\nabla u_{l}(\tau)\right\|^{2}-\left\|\nabla u_{k}(\tau)\right\|^{2}\right) \nabla u_{l}(\tau)\left(\nabla u_{l}-\nabla u_{k}\right) d x d \tau=0, \\
& \lim _{l \rightarrow \infty} \lim _{k \rightarrow \infty} \int_{0}^{T}\left\|\nabla u_{l}(t)\right\|^{2}\left\|\nabla u_{l}(t)-\nabla u_{k}(t)\right\|^{2} d t=0, \\
& \lim _{l \rightarrow \infty} \lim _{k \rightarrow \infty} \int_{0}^{T}\left\|u_{l}(t)-u_{k}(t)\right\|^{2} d t=0, \\
& \lim _{l \rightarrow \infty} \lim _{k \rightarrow \infty} \int_{0}^{T}\left\|\nabla u_{l}(t)-\nabla u_{k}(t)\right\|^{2} d t=0 .
\end{aligned}
$$

Finally, combining (58)-(65) we get $\Phi(\cdot, \cdot) \in C\left(B_{1}\right)$.

\subsection{Existence of global attractor}

Theorem 4.2 Under assumptions (1)-(4), then problems (6)-(9) have a global attractor in $\mathcal{H}$, which is invariant and compact.

Proof Lemma 3.1 and Theorem 4.1 imply the existence of the global attractor.

Acknowledgements

Not applicable.

\section{Funding}

The project is supported by the National Natural Science Foundation of China (Grant No. 11872264, the role of the funding lies in the collection of data, the analysis of the paper and writing the manuscript). 
Competing interests

This paper does not involve conflict of interests between the authors, and all authors declare that they have no competing interests.

\section{Authors' contributions}

This paper is mainly completed by SZ, JZ and HW dealt with the nonlinear damping term as proving the existence of a bounded absorbing set. All authors read and approved the final manuscript.

\section{Author details}

${ }^{1}$ College of Mechanical and Vehicle Engineering, Taiyuan University of Technology, Taiyuan, China. ${ }^{2}$ Department of Mathematics, Taiyuan University of Technology, Taiyuan, China. ${ }^{3}$ School of Applied Science, Taiyuan University of Science and Technology, Taiyuan, China.

\section{Publisher's Note}

Springer Nature remains neutral with regard to jurisdictional claims in published maps and institutional affiliations.

Received: 5 December 2019 Accepted: 14 June 2020 Published online: 22 June 2020

\section{References}

1. Kirchhoff, G.: Vorlesungen über Mechanik. Teubner, Stuttgart (1883)

2. Lazo, P.P.D.: Global solution for a quasilinear wave equations with singular memory. Appl. Math. Comput. 217(19), 7660-7668 (2011)

3. Chueshov, I.: Global attractors for a class of Kirchhoff wave models with a structural nonlinear damping. J. Abstr. Differ. Equ. Appl. 1, 86-106 (2010)

4. Chueshov, I.: Long-time dynamics of Kirchhoff wave models with strong nonlinear damping. J. Differ. Equ. 252 1229-1262 (2012)

5. Yang, Z.J., Wang, Y.Q.: Global attractor for the Kirchhoff type equation with a strong dissipation. J. Differ. Equ. 249 3258-3278 (2010)

6. Yang, Z.J., Ding, P.Y., Liu, Z.M.: Global attractor for the Kirchhoff type equation with strong nonlinear damping and supercritical nonlinearity. Appl. Math. Lett. 33, 12-17 (2014)

7. Ma, Q.Z., Zhong, C.K.: Existence of strong global attractors for hyperbolic equation with linear memory. Appl. Math. Comput. 157, 745-758 (2004)

8. Park, J.R., Kang, J.R.: Global attractor for hyperbolic equation with nonlinear damping and linear memory. Sci. China Ser. A, Math. 53(6), 1531-1539 (2010)

9. Kang, J.R., Rivera, J.M.: Global attractor for an extensible beam equation with localized nonlinear damping and linear memory. Math. Methods Appl. Sci. 34, 1430-1439 (2011)

10. Yang, L., Zhong, C.K.: Global attractor for plate equation with nonlinear damping. Nonlinear Anal. 69, 3802-3810 (2008)

11. Khanmamedov, A.K.: Global attractors for von Karman equations with non-linear interior damping. J. Math. Anal. Appl. 318, 92-101 (2006)

\section{Submit your manuscript to a SpringerOpen ${ }^{\odot}$ journal and benefit from:}

- Convenient online submission

- Rigorous peer review

Open access: articles freely available online

- High visibility within the field

- Retaining the copyright to your article

Submit your next manuscript at $\boldsymbol{\nabla}$ springeropen.com 